\title{
Immunotherapy in tyrosine kinase inhibitor-naïve advanced epidermal growth factor receptor-mutant non-small cell lung cancer-driving down a precarious road in driver-mutated lung cancer
}

\author{
Rebecca Yin Tay ${ }^{1}$, Christoph Jakob Ackermann ${ }^{1}$, Raffaele Califano ${ }^{1,2}$ \\ ${ }^{1}$ Department of Medical Oncology, The Christie NHS Foundation Trust, Manchester, UK; ${ }^{2}$ Division of Cancer Sciences, The University of \\ Manchester, Manchester, UK \\ Correspondence to: Dr Raffaele Califano. Department of Medical Oncology, The Christie NHS Foundation Trust, Wilmslow Road, Manchester M20 \\ 4BX, UK. Email: raffaele.califano@christie.nhs.uk. \\ Comment on: Lisberg A, Cummings A, Goldman JW, et al. A Phase II Study of Pembrolizumab in EGFR-Mutant, PD-L1+, Tyrosine Kinase Inhibitor \\ Naïve Patients With Advanced NSCLC. J Thorac Oncol 2018;13:1138-45.
}

Submitted Sep 12, 2018. Accepted for publication Sep 25, 2018.

doi: $10.21037 /$ tlcr.2018.09.16

View this article at: http://dx.doi.org/10.21037/tlcr.2018.09.16

In patients with advanced non-small cell lung cancer (NSCLC) whose tumour harbour a sensitising epidermal growth factor receptor (EGFR) mutation, international guidelines recommend first-line therapy with an EGFR tyrosine kinase inhibitor (TKI) due to longer progression free survival (PFS), higher overall response rate (ORR) and improved quality of life compared to platinum doublet chemotherapy (1,2). Eventually, most patients develop resistance to initial EGFR-TKI therapy. The most common resistance mechanism in approximately $60 \%$ of patients is an acquired threonine-to-methionine amino acid substitution at position 790 (T790M) of the EGFR kinase domain in exon 20 resulting in reduced adenosine triphosphate (ATP) competitive TKI-binding that confers loss of drug activity (3). Third-generation EGFR-TKI osimertinib has shown to overcome T790M-induced drug resistance with a significantly improved PFS [10.1 vs. 4.4 months, hazard ratio (HR) $0.3, \mathrm{P}<0.001]$ and ORR [71\% vs. 31\%, odds ratio (OR) 5.39, $\mathrm{P}<0.001]$ compared to platinum-pemetrexed chemotherapy (4). Osimertinib has also been recently approved by the Food and Drug Administration (FDA) - and the European Medicines Agency (EMA) - as first-line therapy for EGFR-mutant (mt) patients due to longer PFS (18.9 vs. 10.2 months, HR 0.46, $\mathrm{P}<0.001)$ compared to first-generation EGFR-TKIs (5).

Anti-PD-(L)1 drugs such as nivolumab, pembrolizumab and atezolizumab are now standard of care for pre-treated advanced NSCLC patients due improved median overall survival (OS) when compared to docetaxel (6-9). Notably, these studies enrolled EGFR-mt and ALK positive patients who had progressed on prior TKI therapy. In the first-line setting, pembrolizumab is approved as a single agent for the treatment of patients with metastatic NSCLC whose tumors have high programmed death-ligand 1 (PD-L1) expression $(\geq 50 \%)$ and in combination with pemetrexed and carboplatin, for unselected patients with metastatic nonsquamous NSCLC $(10,11)$. The current license excludes patients with EGFR or ALK genomic tumour aberrations given these patients were not enrolled in the registration studies.

As these pivotal first-line checkpoint inhibitor (CPI) trials excluded EGFR-mt patients, the efficacy of CPI in the first-line, particularly prior to EGFR-TKI exposure remains unclear. This is the question that Lisberg et al. aimed to address in their phase II study of first-line pembrolizumab in TKI naïve EGFR-mt advanced NSCLC with a PD-L1 tumour proportion score (TPS) $\geq 1 \%$ now reported in the Fournal of Thoracic Oncology (12). This single institution study included patients with either a sensitising or non-sensitising EGFR mutation. The study failed to meet its primary end point of ORR $\geq 26 \%$ and was closed early due to futility, with 11 out of 25 patients enrolled. Ten 
patients were evaluable for response ( $\mathrm{n}=1$ excluded due to an EGFR-mutation reporting error). The ORR was $0 \%$ and stable disease was achieved in 7 (70\%) patients. Median PFS was short (119 days) with median OS not yet reached. The rate of treatment-related adverse events (TRAEs) was $46 \%$ with one patient experiencing grade 3 treatmentrelated transaminitis resulting in treatment discontinuation. No grade 4-5 TRAEs were reported.

Although the small population and early termination of the trial limits definitive conclusions, the findings from this study support the increasing body of evidence that point to limited benefit of CPI monotherapy in EGFR-mt NSCLC. A meta-analysis by Lee $e t$ al. assessed the benefit of secondline programmed death-1 (PD-1)/PD-L1 monotherapy (pembrolizumab, nivolumab, atezolizumab) compared to docetaxel in the EGFR-mt defined subgroups. Whilst a $33 \%$ risk reduction for death in the EGFR-wild type subgroup was detected, there was no survival advantage in the EGFR-mt population with CPI. In the pre-treated NSCLC cohort within the phase 1b KEYNOTE-001 study, EGFR-mt NSCLC was associated with worse median OS compared to EGFR-wild type NSCLC (13). Gainor et al. also reported a retrospective series showing poor ORR (4.5\%) to PD-(L)1 blockade in pre-treated EGFR-mt patients (14). Similarly, Lisberg et al. report a notable lack of objective response to pembrolizumab, even within a PD-L1 positive enriched study population that included 7 patients (70\%) with high ( $\geq 50 \%)$ PD-L1 expression. In contrast to the $27.3 \%$ ORR achieved with first-line pembrolizumab in an EGFR wild type NSCLC population expressing PD-L1 TPS $\geq 1 \%$ (15), no patient in this phase 2 study achieved partial or complete response (ORR 0\%) suggesting overall low intrinsic tumour immunogenicity in EGFR-mt lung cancer.

A number of factors may account for reduced response rates and survival to CPI in EGFR-mt NSCLC. Firstly, an uninflamed or 'immune-cold' tumour microenvironment is observed in EGFR-mt lung tumours with low levels of T-cell infiltration and proportion of PD-L1+/CD8+ tumour infiltrating lymphocytes (TILs) (16). CD73 overexpression resulting in a reduced IFN gamma signature observed in EGFR-mt NSCLC may also contribute to a resistant immunophenotype (17). Tumour mutation burden (TMB) has been studied as a predictive biomarker independent of PD-L1 expression in NSCLC with high TMB linked to improved CPI response. Cigarette smoking induced carcinogenesis is associated with neoantigen formation and high tumour mutational load, a genetic phenotype in contrast to EGFR-mt NSCLC where a much lower TMB is observed compared to NSCLC without a driver mutation (18). Resistance to EGFR-TKI therapy has shown to result in increased TMB in a retrospective series that further supports the sequencing of EGFR-TKIs prior to CPI (19).

Lisberg et al. report rates of TRAEs similar to previous pembrolizumab monotherapy studies however without the same magnitude of clinical benefit. One patient developed adrenal insufficiency on study, necessitating lifelong steroid replacement; a morbidity that may have been delayed by earlier TKI initiation. Nine patients on study received subsequent treatment following progression on pembrolizumab including TKI therapy in 6 patients with an EGFR sensitising mutation. One patient developed grade 5 pneumonitis and subsequently died of respiratory failure 3 months after initiation of erlotinib and more than 4 months after the last dose of pembrolizumab. Whilst pneumonitis was attributed to erlotinib, the impact of prior pembrolizumab exposure cannot be excluded. Due to the short median follow up time, it is unclear if delay in the initiation of TKI therapy resulted in inferior response rates, survival or if prior exposure to pembrolizumab increases risk of TKI-induced pulmonary toxicity.

The results of the trial by Lisberg et al. support current guidelines that advise administration of EGFR-TKI therapy prior to immunotherapy in advanced NSCLC harbouring EGFR sensitising mutations. Notably, this study also enrolled 3 patients with non-sensitising EGFR mutations and high PD-L1 expression ( $>50 \%)$. In this subgroup, firstline pembrolizumab resulted in poor clinical benefit. Two patients reported early disease progression and 1 patient experiencing severe TRAEs leading to study discontinuation with only a short 6-week period of disease control before radiologic progression. These outcomes suggest platinumdoublet chemotherapy remains the most appropriate firstline option in non-sensitising EGFR-mt lung cancer irrespective of PD-L1 status.

A number of combination strategies seek to improve CPI response and survival benefit in EGFR-mt NSCLC. Trials that combine an anti-PD-(L)1 agent with an EGFR-TKI have shown encouraging clinical activity, however remain investigational due to high rates of TRAEs (20). A more promising approach may be use of CPI after progression on EGFR-TKIs in combination with other agents. A preplanned subgroup analysis of IMPOWER 150, evaluating EGFR and ALK positive patients (13\% of intention-totreat population), showed that the addition of atezolizumab 
to carboplatin/paclitaxel/bevacizumab (CPB) resulted in longer OS (HR 0.54) compared to CPB alone (21).

In the context of previous data, the prospective trial by Lisberg et al. serves as a timely reminder of the distinct differences in intrinsic tumour biology between oncogenicdriven EGFR-mt tumours and EGFR-wt lung cancer and reiterates the modest efficacy of CPI in EGFR-mt NSCLC patients, even in presence of high PD-L1 expression.

\section{Acknowledgements}

None.

\section{Footnote}

Conflicts of Interest: The authors have no conflicts of interest to declare.

\section{References}

1. Novello S, Barlesi F, Califano R, et al. Metastatic nonsmall-cell lung cancer: ESMO Clinical Practice Guidelines for diagnosis, treatment and follow-up. Ann Oncol 2016;27:v1-27.

2. Hanna N, Johnson D, Temin S, et al. Systemic Therapy for Stage IV Non-Small-Cell Lung Cancer: American Society of Clinical Oncology Clinical Practice Guideline Update. J Clin Oncol 2017;35:3484-515.

3. Ma C, Wei S, Song Y. T790M and acquired resistance of EGFR TKI: a literature review of clinical reports. J Thorac Dis 2011;3:10-8.

4. Mok TS, Wu YL, Ahn MJ, et al. Osimertinib or PlatinumPemetrexed in EGFR T790M-Positive Lung Cancer. N Engl J Med 2017;376:629-40.

5. Soria JC, Ohe Y, Vansteenkiste J, et al. Osimertinib in Untreated EGFR-Mutated Advanced Non-Small-Cell Lung Cancer. N Engl J Med 2018;378:113-25.

6. Borghaei H, Paz-Ares L, Horn L, et al. Nivolumab versus Docetaxel in Advanced Nonsquamous Non-Small-Cell Lung Cancer. N Engl J Med 2015;373:1627-39.

7. Brahmer J, Reckamp KL, Baas $P$, et al. Nivolumab versus Docetaxel in Advanced Squamous-Cell Non-Small-Cell Lung Cancer. N Engl J Med 2015;373:123-35.

8. Herbst RS, Baas P, Kim DW, et al. Pembrolizumab versus docetaxel for previously treated, PD-L1-positive, advanced non-small-cell lung cancer (KEYNOTE-010): a randomised controlled trial. Lancet 2016;387:1540-50.

9. Rittmeyer A, Barlesi F, Waterkamp D, et al. Atezolizumab versus docetaxel in patients with previously treated non-small-cell lung cancer (OAK): a phase 3, openlabel, multicentre randomised controlled trial. Lancet 2017;389:255-65.

10. Reck M, Rodríguez-Abreu D, Robinson AG, et al. Pembrolizumab versus Chemotherapy for PD-L1Positive Non-Small-Cell Lung Cancer. N Engl J Med 2016;375:1823-33.

11. Gandhi L, Rodríguez-Abreu D, Gadgeel S, et al. Pembrolizumab plus Chemotherapy in Metastatic Non-Small-Cell Lung Cancer. N Engl J Med 2018;378:2078-92.

12. Lisberg A, Cummings A, Goldman JW, et al. A Phase II Study of Pembrolizumab in EGFR-Mutant, PD-L1+, Tyrosine Kinase Inhibitor Naive Patients With Advanced NSCLC. J Thorac Oncol 2018;13:1138-45.

13. Hui R, Gandhi L, Carcenery Costa E. Long-term OS for patients with advanced NSCLC enrolled in the KEYNOTE-001 study of pembrolizumab (Abstract). J Clin Oncol 2016;43:9026.

14. Gainor JF, Shaw AT, Sequist LV, et al. EGFR Mutations and ALK Rearrangements Are Associated with Low Response Rates to PD-1 Pathway Blockade in Non-Small Cell Lung Cancer (NSCLC): A Retrospective Analysis. Clin Cancer Res 2016;22:4585-93.

15. Lopes G, Wu YL, Kudaba I, et al. Pembrolizumab versus platinum-based chemotherapy as first-line therapy for advanced/metastatic NSCLC with a PDL1 tumor proportion score (TPS) $\geq 1 \%$ : Openlabel, phase 3 KEYNOTE-042 study. J Clin Oncol 2018;36:LBA4.

16. Soo RA, Lim SM, Syn NL, et al. Immune checkpoint inhibitors in epidermal growth factor receptor mutant non-small cell lung cancer: Current controversies and future directions. Lung Cancer 2018;115:12-20.

17. Streicher K, Higgs BW, Wu S, et al. Increased CD73 and reduced IFNG signature expression in relation to response rates to anti-PD-1(L1) therapies in EGFR-mutant NSCLC. J Clin Oncol 2017;35:11505.

18. Schrock A, Sharma N, Peled N, et al. MA14.01 Updated Dataset Assessing Tumor Mutation Burden (TMB) as a Biomarker for Response to PD-1/PD-L1 Targeted Therapies in Lung Cancer (LC). J Thorac Oncol 2017;12:S422.

19. Offin M, Rizvi H, Tenet M, et al. Tumor Mutation Burden and Efficacy of EGFR-Tyrosine Kinase Inhibitors in Patients with EGFR-Mutant Lung Cancers. Clin Cancer Res 2018. [Epub ahead of print]. 
20. Ahn MJ, Sun JM, Lee SH, et al. EGFR TKI combination with immunotherapy in non-small cell lung cancer. Expert Opin Drug Saf 2017;16:465-9.

Cite this article as: Tay RY, Ackermann CJ, Califano R. Immunotherapy in tyrosine kinase inhibitor-naïve advanced epidermal growth factor receptor-mutant non-small cell lung cancer-driving down a precarious road in driver-mutated lung cancer. Transl Lung Cancer Res 2018;7(Suppl 4):S377-S380. doi: 10.21037/tlcr.2018.09.16
21. Socinski MA, Jotte RM, Cappuzzo F, et al. Atezolizumab for First-Line Treatment of Metastatic Nonsquamous NSCLC. N Engl J Med 2018;378:2288-301. 